\title{
Detection of angiospastic disorders in the microcirculatory bed using laser diagnostics technologies
}

\author{
Irina N. Makovik* ${ }^{* \dagger}$, Andrey V. Dunaev*, Victor V. Dremin*, \\ Alexander I. Krupatkin ${ }^{\dagger}$, Viktor V. Sidorov ${ }^{\star}$, Lyudmila S. Khakhicheva ${ }^{\S}$, \\ Vadim F. Muradyan ${ }^{\S}$, Olga V. Pilipenko*, Ilya E. Rafailov \\ and Karina S. Litvinova ${ }^{\|, * *}$ \\ *Biomedical Photonics Instrumentation Group \\ Scientific-Educational Centre of "Biomedical Engineering" \\ Orel State University named after I. S. Turgenev \\ Orel 302026, Russian Federation \\ ${ }^{\dagger}$ Priorov Central Research Institute of Traumatology and Orthopaedics \\ Moscow 127299, Russian Federation \\ "SPE "LAZMA" Ltd, Moscow 125252, Russian Federation \\ $\S_{\text {Orel Regional Clinical Hospital }}$ \\ Orel 302028, Russian Federation \\ "School of Engineering and Applied Sciences \\ Aston Institute of Photonic Technologies \\ Aston University, Birmingham, B4 7ET, UK \\ "Optoelectronics and Biomedical Photonics Group \\ Aston Institute of Photonic Technologies \\ Aston University, Birmingham, B4 7ET, UK \\ **Aston Medical School \\ Aston University, Birmingham, B4 7ET, UK \\ †irina.makovik@gmail.com
}

Received 19 December 2016

Accepted 23 April 2017

Published 9 June 2017

\begin{abstract}
The evaluation of the microcirculatory bed functional state and the identification of angiospastic disorders with related complications, when the pathological changes are reversible, have an important role in medical practice. The aim of this study was to evaluate the possibility of using optical noninvasive methods and the cold pressor test to solve this problem.
\end{abstract}

$\dagger$ Corresponding author.

This is an Open Access article published by World Scientific Publishing Company. It is distributed under the terms of the Creative Commons Attribution 4.0 (CC-BY) License. Further distribution of this work is permitted, provided the original work is properly cited. 


\section{N. Makovik et al.}

A total of 33 patients with rheumatological diseases and 32 healthy volunteers were included in the study. Laser Doppler flowmetry, tissue reflectance oximetry and pulse oximetry were used as optical noninvasive methods. The parameters were recorded before, immediately after and 20 min after the cold pressor test. Based on the measured parameters, the complex parameters of the microcirculatory bed were calculated.

A detailed statistical analysis of the parameter changes for each individual in the two groups displayed diverse microcirculatory bed parameter responses upon cold exposure, with differing recovery of parameters after CPT. New diagnostic criteria were proposed for the identification of angiospastic disorders. According to the proposed criteria, 27 people of the volunteers group were confirmed to not display any disorders. In the patient group, however, 18 people were observed to have a relatively normal functional state of the microcirculatory bed, while 15 people were observed to have a possible tendency to angiospasm. To highlight the differences between a relatively normal state and presence of angiospastic disorders, statistical analysis of experimental data was carried out, which revealed significant differences. Further analysis of data with angiospastic disorders identified a relationship between their diagnoses and the results of laboratory studies.

Thus, the evaluation of combined noninvasive optical diagnostic method use, the cold pressor test and proposed diagnostic criteria showed a positive result. This approach can be used to detect the presence of possible angiospastic disorders and related complications, as well as microcirculatory bed disorders against the background of other diseases.

Keywords: Noninvasive diagnostics; laser Doppler flowmetry; tissue reflectance oximetry; pulse oximetry; cold pressor test; microcirculatory bed; angiospasm.

\section{Introduction}

The evaluation of the microcirculatory bed functional state in the upper limbs presents itself as a major problem in modern medical diagnostics. Its essence lies in the development and improvement of diagnostic methods, which would contribute to a better definition of reserve and adaptive capabilities of the microcirculatory bed, as well as the timely detection of various pathologies.

Angiospastic disorders are one such pathology. These disorders provoke vasospasm of vessels and represent a reversible localized or diffuse vasoconstriction of arteries or smaller blood vessels. ${ }^{1}$ Today, there are a distinguished number of diseases of the angiospastic nature, such as Raynaud's disease, acrocyanosis, and livedo reticularis. ${ }^{2}$

These disorders may be in the form of angiospastic attack with the occurrence of reversible ischemia, without compromising the integrity of the vascular system and tissues. Alternatively, they may take the form of prolonged ischemia, which result in the deterioration of tissue blood supply in addition to an observed imbalance of major skin chromophore concentrations (oxyhaemoglobin and deoxyhaemoglobin), which will eventually give rise to hypoxia of membranes, development of tissue swelling and the appearance of necrobiotic processes. $^{3-5}$ Thus, the expression of data processes will increase with an increase of the interval between tissue oxygen demand and the level of blood flow.

Angiospasm plays an important role in the pathogenesis of a variety of diseases. It can appear as an independent disease, ${ }^{6}$ appear under the influence of external factors, various chemicals and medicinal drugs, ${ }^{7}$ and can develop in the background of such diseases as scleroderma, ${ }^{8,9}$ systemic lupus erythematosus ${ }^{10,11}$ and rheumatoid arthritis. ${ }^{12,13}$ Involvement of the vessels into the pathological process, in the background of existing connective tissue system disorganizations can lead to even greater aggravation of the disease course and outcome and the development of tissue hypoxia. This, in most cases, will influence the approach to treatment and prognosis of the disease as a whole.

Thus, a timely detection of angiospastic microcirculatory bed disorders as well as tissue oxygen supply and consumption changes (transcapillary exchange) is important, both in the manifestation of their primary characteristics (when pathological changes are still reversible) and in various 
pathologies, in order to prevent aggravation of diseases and reach better treatment.

Certain optical noninvasive technologies, namely the methods of laser Doppler flowmetry (LDF), ${ }^{14,15}$ tissue reflectance oximetry (TRO) ${ }^{16}$ and pulse oximetry (PO), ${ }^{17}$ appear to have potential for the study of the microcirculatory bed functional state and the detection of angiospastic disorders. These methods allow for in vivo evaluation of the peripheral circulation level, transport dynamics, the value of blood oxygen saturation in the microvessels and the percentage of oxyhaemoglobin in arterial blood.

Furthermore, the above methods are capable of providing vast amounts of physiological data. This data comes in the form of frequency ranges $(0.0095-$ $1.6 \mathrm{~Hz})$, which reflect the different mechanisms of regulation. There are currently five categorized frequency ranges. ${ }^{18}$ The pulse $(0.8-1.6 \mathrm{~Hz})$ and respiratory $(0.2-0.4 \mathrm{~Hz})$ ranges are identified among the passive mechanisms of regulation, which characterize the influence of heart rate and movement of the thorax. ${ }^{18-20}$ Active mechanisms of regulation are myogenic $(0.047-0.145 \mathrm{~Hz})$, transmitting the activity of vascular smooth muscle cells, ${ }^{21-23}$ neurogenic $(0.021-0.046 \mathrm{~Hz})$, showing the influence of neurogenic sympathetic vasomotor activity, ${ }^{21,24}$ and endothelial $(0.0095-0.02 \mathrm{~Hz})$, reflecting the vasomotor activity of vascular endothelium. ${ }^{25-27}$

Thus, with the use of this mathematical apparatus, it possible to evaluate the main factors provoking vasoconstriction and angiospastic disorder. Analysis of endothelial fluctuations reveals the presence of disturbances in the vascular endothelial function. ${ }^{28}$ As is known, these violations are manifested in the form of a decrease in the concentration of endogenous vasodilators acting on the endothelium, ${ }^{29-32}$ namely, in reduced synthesis of $\mathrm{NO},{ }^{33,34}$ and also in an increase of vasoconstrictor substance activation, such as endothelin-1. ${ }^{35,36}$ According to the analysis of oscillation mechanisms of neurogenic and myogenic genesis, it is possible to judge the presence of other factors, which induce angiospastic disorders, such us activation of the alpha-adrenoceptor, ${ }^{37}$ increases in smooth muscle contractility and about launching of different vasomotor reflexes in the result of activation of the sympathetic nervous system. . $^{6,38}$

In this regard, evaluation of the prospective combined use of these methods was proposed, in order to analyze all the changes arising from angiospasm; beginning with blood flow violations and ending with the development of hypoxia.

The combined application of the suggested diagnostic methods and various functional tests contributes to the increased overall level of informative diagnostic data. Their use contributes to the evaluation of not only the general condition of the microcirculatory bed, but its reserve and adaptive capacities. One provocative test, used in the study of the human body's functional state, is the cold pressor test (CPT). This test is carried out through complete immersion of the upper or lower limbs of the body in a container with cold water. The water temperature during the experiments may be from $0^{\circ} \mathrm{C}$ to $15^{\circ} \mathrm{C}$, with study durations reaching up to 30 min. ${ }^{39}$ The stimulus, which in the case of the CPT immersing in cold water, provokes many interrelated processes. Namely, these are the changes in blood pressure and heart rate, ${ }^{40-42}$ irritation of thermoreceptors, activation of the sympathetic nervous system, ${ }^{43,44}$ constriction of muscle containing vessels, ${ }^{45}$ synthesis stimulation of various neurotransmitters (such as noradrenaline, dopamine $^{46}$ ), etc. After the termination of cold exposure in a normal functional state of the body and the absence of disorders, there is a stabilization and recovery of all body processes. Presence of these disorders exhibits no such recovery.

This test, widely used in clinical practice and in combination with various diagnostic methods, is used for additional research and prediction of nervous and cardiovascular system disorders as well as aiding in the identification of such conditions as Alzheimer's disease, hypertension, glaucoma, and others. ${ }^{47-49}$

Thereby, the aim of this study was to evaluate the possibility of combining the LDF, TRO and PO methods with the cold pressor test for the analysis of reserve and adaptive abilities of the microcirculatory bed in the upper limbs and detecting the presence of angiospastic disorders.

\section{Materials and Methods}

\subsection{Inclusion and exclusion criteria}

To evaluate the combined use functionality of the LDF, tissue reflectance oximetry, pulse oximetry and cold pressor test methods, experimental studies were conducted on 32 healthy volunteers and 
33 patients from the Rheumatology Department of the Orel Regional Clinical Hospital (Orel, Russia).

The study was approved by the local Committee for Human Biomedical Research Ethics and was carried out in accordance with the principles outlined in the 2002 Declaration of Helsinki by the World Medical Association. The study protocol and its purpose were explained in detail to each subject and the informed consent was obtained from all of the subjects.

The group of healthy volunteers included 16 men (average age $-22 \pm 1$ year) and 16 women (mean age $-22 \pm 2$ years). The participating volunteer group was collected through an internal university advertisement circulated by physical posters and notification email. According to the preliminary interview, these people did not exhibit disorders of the cardiovascular system and led healthy lifestyles.

The group of patients includes individuals with the following diseases: rheumatoid arthritis $(n=16)$, psoriatic arthritis $(n=2)$, gout $(n=3)$, systemic lupus erythematosus $(n=3)$, systemic scleroderma $(n=2)$, Primary gonarthrosis, bilateral $(n=4)$, spondylitis seronegative $(n=1)$, chronic rheumatic heart disease $(n=1)$, ankylosing spondylitis $(n=1)$.

Selection of patient volunteers was assisted in by the attending physician, based on the medical history, analysis of the disease and any co-morbidities, results of prior studies conducted using different diagnostics methods and in the presence of laboratory blood test changes (increases in laboratory parameters such as rheumatoid factor, antinuclear factors, thrombocytosis and anaemia). Additionally, patient complaints about pain in their fingers, increased sensitivity to cold and cold induced discoloration were also taken into account.

The main characteristics of the patient group are presented in Table 1. The data-range averages, as well as the minimum and maximum values, are provided for each parameter.

As described previously, disorders of upper limb microcirculatory bed are most commonly found as one of the forms of rheumatological profile disease pathologies. These diseases are more common in the elderly. It is thus necessary to clearly differentiate between a healthy state and one with microcirculatory bed disorders. A group of healthy young volunteers was recruited as a control to ensure an "extreme" state of good health, as they would
Table 1. The main characteristics of the patient group.

\begin{tabular}{ll}
\hline Characteristics & Rheumatology subjects \\
\hline Sex, M/F & $8 / 25$ \\
Age M/F, y & $36 \pm 12 / 61 \pm 13$ \\
Disorder duration, y & $9(0.08-30)$ \\
Systolic BP, mmHg & $131.9(110-160)$ \\
Diastolic BP, mmHg & $74.1(70-90)$ \\
Pulse $/ \mathrm{min}$ & $82.3(56-84)$ \\
RF, IU/ml & $55.9(5-512)$ \\
CRP, mg/l & $41.6(0.8-192)$ \\
RBC, $10^{12} / 1$ & $4.3(3.5-5.1)$ \\
HGB, g/l & $126.2(91-155)$ \\
MCHC & $0.88(0.7-0.9)$ \\
PLT, $10^{9} / 1$ & $276.8(118-457)$ \\
WBC, $10^{9} / 1$ & $7.6(4.7-8.7)$ \\
LYM, $\%$ & $30.6(5-65)$ \\
MONO, $\%$ & $3.8(1-9)$ \\
ESR, mm/h & $25.9(4-62)$ \\
\hline
\end{tabular}

Notes: RF — rheumatoid factor; CRP - C-reactive protein; RBC — red blood cells; HGB — haemoglobin; MCHC — mean corpuscular haemoglobin concentration; PLT - platelets; WBC — white blood cells; LYM - lymphocyte; MONO monocyte; ESR - erythrocyte sedimentation rate.

present the lowest chance of exhibiting any undesired physiological conditions.

\subsection{Study protocol}

The laser analyzer of blood microcirculation "LAKK-OP" was used to register parameters of the microcirculatory bed on healthy volunteers. The multifunctional laser diagnostic complex "LAKK-M" (SPE "LAZMA" Ltd, Russia) was similarly employed on the patients. These diagnostic devices utilized identical measurement channels and are designed to study the state of peripheral blood flow. This is achieved by a simultaneous radiation of sample tissue through superficial contact with optical sensors. A $1064 \mathrm{~nm}$ laser wavelength for LDF and $532 \mathrm{~nm}$ and $635 \mathrm{~nm}$ radiation wavelengths for the TRO and pulse oximetry methods were delivered and received by the optical sensors. The location of these sensors on the fingers during the experimental studies on volunteers using the "LAKK-OP" is presented in Fig. 1(a); the experimental setup for the study on patients using the "LAKK-M" is presented in Fig. 1(b).

The LDF 3.0.2.384 and LDF 3.1.1.407 software packages were used for the frequency analysis of the passive and active microcirculatory regulation mechanisms. These software packages use a 


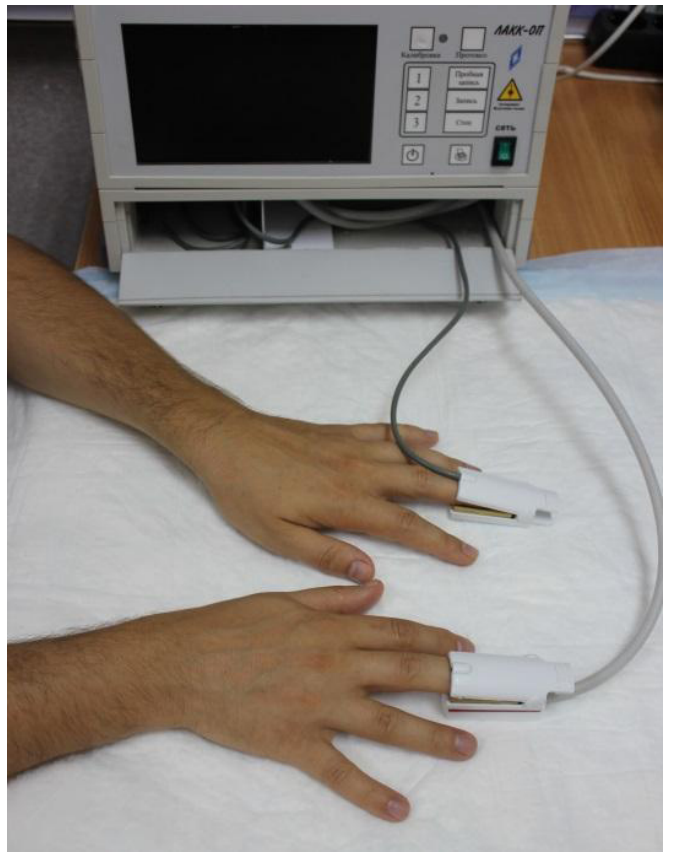

(a)

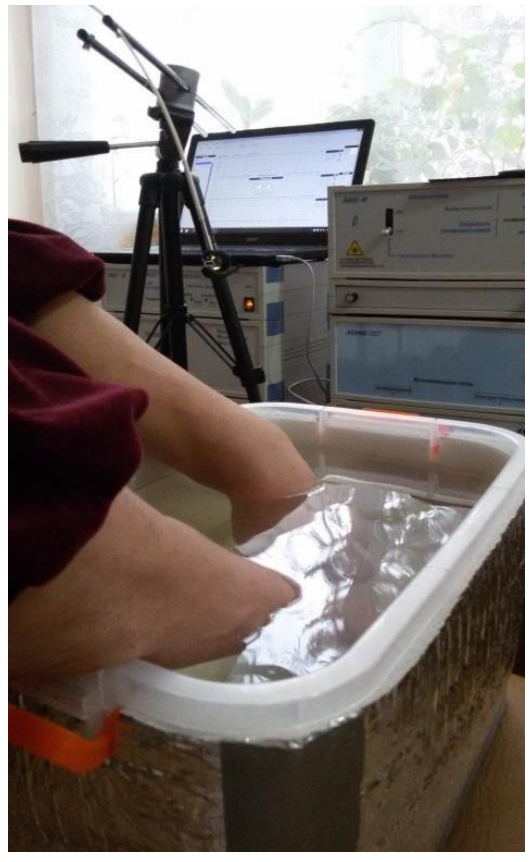

(b)

Fig. 1. The location of the optical sensors on the fingers during the experimental studies on volunteers using the "LAKK-OP" (a), the experimental setup for the study on patients using the "LAKK-M" (b).

continuous wavelet transform, with the Morle complex valued wavelet being used as the analyzing wavelet. ${ }^{50,51}$

Three basic (background) tests (BT), 5 min duration each, were involved in the experiment using the CPT: BT1 was recorded before CPT, BT2 immediately after and BT3 20 min after CPT completion. The temperature of water during the CPT was $14.8 \pm 0.2^{\circ} \mathrm{C}$. A contactless electronic thermometer "Sensitec NB401" was used for the temperature control of water and volunteer skin. The LAKK device measurements were performed on the skin pad (palmar surface) of the right middle finger. This area was chosen because it is rich in arteriolarvenular anastomoses (AVA), which depend on the sympathetic innervation as well as autonomic and sensory nerve fibers. Analyzes of the changes of parameters in this area allow to diagnose various microvascular disorders, which begin at the level of the microcirculatory bed of the fingers, including angiospastic disorders. Moreover, the area with AVA has lower spatial variability due to a low disparity in skin capillary density, which ensures lower variability of parameters and better reproducibility of data. ${ }^{52,53}$

All measurements were performed in conditions of physical and mental rest $2 \mathrm{~h}$ after a meal.
Volunteers also underwent a preliminary adaptation to room temperature $24-25^{\circ} \mathrm{C}$ for $15-20 \mathrm{~min}$ in a sitting position, with the right arm on the table at heart level. The adaptation of volunteers to standard room temperature and abidance of study protocol during all measurements reduces the influence of different factors on results of diagnosis.

\section{3. $\quad$ Measured and calculated parameters}

Basic microcirculatory bed parameters were registered during experimental studies, providing a vast array of information. The changes in blood flow (blood perfusion of the tissue) per unit time in the probed volume of the tissue was provided by the index of blood microcirculation $\left(I_{m}\right)$. Measurement of tissue oxygen saturation $\left(\mathrm{S}_{t} \mathrm{O}_{2}\right)$ described the percentage of oxyhaemoglobin on the background of the sum of all haemoglobin fractions (oxyhaemoglobin and deoxyhaemoglobin). The relative volume fractions of haemoglobin (blood volume) in a test volume of biological tissue and the percentage of oxyhaemoglobin in arterial blood are provided by the measured relative blood volume $\left(V_{b}\right)$ and arterial oxygen saturation $\left(\mathrm{S}_{a} \mathrm{O}_{2}\right)$, respectively. 


\section{N. Makovik et al.}

Due to the expediency of the complex diagnostic of the upper limb microcirculatory bed, the oxygen extraction (OE) and the rate of oxygen consumption (OC) were calculated based on the measured parameters obtained by a methodology relying on the analysis of LDF and TRO determined amplitude-frequency spectra. ${ }^{54,55}$ The calculation of these parameters makes it possible to evaluate the changes in the tissue oxygen supply and consumption. In this case, the value of the OE represents the process of oxygen diffusion from the arterial blood into the tissue. The calculation of the rate of OC gives an indication of the dynamics of oxygen utilization by tissues.

$$
\mathrm{OE}=\left(\mathrm{S}_{a} \mathrm{O}_{2}-\mathrm{S}_{v} \mathrm{O}_{2}\right) / \mathrm{S}_{a} \mathrm{O}_{2},
$$

where $\mathrm{S}_{v} \mathrm{O}_{2}$ is venous blood oxygen saturation.

To determine the values of venous blood oxygen saturation, the amplitude of oscillations associated with passive mechanisms of $\mathrm{S}_{t} \mathrm{O}_{2}$ regulation were analyzed, namely: the amplitudes of the cardiac A $\left(\mathrm{S}_{t} \mathrm{O}_{2}\right)_{c}$ and respiratory $\mathrm{A}\left(\mathrm{S}_{t} \mathrm{O}_{2}\right)_{r}$ oscillations.

$$
A\left(\mathrm{~S}_{t} \mathrm{O}_{2}\right)_{c} / A\left(\mathrm{~S}_{t} O_{2}\right)_{r}>1,
$$

If the condition is met, then the venous blood oxygen saturation is calculated as follows:

$$
\mathrm{S}_{v} \mathrm{O}_{2}=\frac{\mathrm{S}_{t} \mathrm{O}_{2}}{A\left(\mathrm{~S}_{t} \mathrm{O}_{2}\right)_{c} / A\left(\mathrm{~S}_{t} \mathrm{O}_{2}\right)_{r}},
$$

where $\mathrm{A}\left(\mathrm{S}_{t} \mathrm{O}_{2}\right)_{c}, \mathrm{~A}\left(\mathrm{~S}_{t} \mathrm{O}_{2}\right)_{r}$ are the maximum oscillation amplitudes of cardiac and respiratory origin of the tissue oxygen saturation.

This variant is predominant in most cases recorded on skin with AVA's.

In the case of the resonance of tissue oxygen saturation oscillations, when high-amplitude oscillations are observed only in one of the active regulatory mechanisms against a sharp suppression of oscillations and a decrease of amplitude in the other ranges, the calculation of $\mathrm{S}_{v} \mathrm{O}_{2}$ has several features. In the case of the oscillation resonance in the general myogenic or respiratory ranges, the venous blood oxygen saturation is calculated as follows:

$$
\mathrm{S}_{v} \mathrm{O}_{2}=\frac{\mathrm{S}_{t} \mathrm{O}_{2}}{\mathrm{BI}\left(\mathrm{S}_{t} \mathrm{O}_{2}\right)}
$$

where $\mathrm{BI}\left(\mathrm{S}_{t} \mathrm{O}_{2}\right)$ is bypass index, is calculated using the $\mathrm{S}_{t} \mathrm{O}_{2}$-gram:

$$
\mathrm{BI}\left(\mathrm{S}_{t} \mathrm{O}_{2}\right)=1+\frac{A\left(\mathrm{~S}_{t} \mathrm{O}_{2}\right)_{n}}{A\left(\mathrm{~S}_{t} \mathrm{O}_{2}\right)_{m}}
$$

where $A\left(\mathrm{~S}_{t} \mathrm{O}_{2}\right)_{n}, A\left(\mathrm{~S}_{t} \mathrm{O}_{2}\right)_{m}$ are the amplitudes of the oscillations of the tissue oxygen saturation in neurogenic and myogenic ranges.

The rate of OC is calculated based on the analysis of the LDF-gram oscillations and the previously calculated value of venous blood oxygen saturation:

$$
\mathrm{OC}=I_{m \text { nutr }} \times\left(\mathrm{S}_{a} \mathrm{O}_{2}-\mathrm{S}_{v} \mathrm{O}_{2}\right),
$$

where $I_{m \text { nutr }}$ is the proportion of nutritive blood flow in the general microcirculation (nutritive blood flow). This value characterizes the blood flow in the capillaries and is calculated by the formula:

$$
I_{m \text { nutr }}=I_{m} / \mathrm{BI}\left(I_{m}\right),
$$

where $\operatorname{BI}\left(I_{m}\right)$ is the bypass index, characterizing blood flow in the AVA. This parameter is calculated using the LDF-gram and the formula:

$$
\mathrm{BI}\left(I_{m}\right)=\mathrm{BI}\left(I_{m}\right)_{1}+\mathrm{BI}\left(I_{m}\right)_{2} .
$$

The component $\operatorname{BI}\left(I_{m}\right)_{1}$ is calculated by formula (5) using perfusion data $\left(I_{m}\right)$. In the case of dominance of blood flow oscillations, associated with endothelial regulation mechanisms in the calculation, the oscillation amplitude of neurogenic origin $\left(A_{n}\right)$ is replaced by the amplitude of oscillation associated with endothelial mechanisms $\left(A_{e}\right)$ during calculation of the bypass index.

$\mathrm{BI}\left(I_{m}\right)_{2}$ is calculated by the formula:

$$
\mathrm{BI}\left(I_{m}\right)_{2}=A\left(I_{m}\right)_{\mathrm{pass}} / A\left(I_{m}\right)_{m},
$$

where $A\left(I_{m}\right)_{\text {pass }}$ is the maximum oscillation amplitude of blood flow associated with passive mechanisms of regulation (oscillation of cardiac or respiratory origin).

$\mathrm{BI}\left(I_{m}\right)_{2}$ is taken into account in the calculation of bypass index if $\mathrm{BI}\left(I_{m}\right)_{2} \geq 1 .{ }^{54}$

Thus, the calculation of these parameters makes it possible to assess the perfusion on nutritive (capillaries) and shunt (AVA)-based blood flow pathways of the microcirculatory bed.

To estimate the oscillatory component of microvascular tone, the values of endothelial (ET), neurogenic (NT) and myogenic (MT) tone were determined.

$$
\mathrm{ET}=\sigma / A\left(I_{m}\right)_{e},
$$

where $\sigma$ is mean square deviation of the blood microcirculation index $\left(I_{m}\right) ; A\left(I_{m}\right)_{e}$ - the greatest value of oscillation amplitude of perfusion in the endothelial range. 


$$
\mathrm{NT}=\sigma / A\left(I_{m}\right)_{n}
$$

where $A\left(I_{m}\right)_{n}$ is the greatest value of oscillation amplitude of perfusion in the neurogenic range.

$$
\mathrm{MT}=\sigma / A\left(I_{m}\right)_{m},
$$

where $A\left(I_{m}\right)_{m}$ is the greatest value of oscillation amplitude of perfusion in the myogenic range.

In addition to analyze the oscillatory component of microvascular tone, the calculated endothelial and neurogenic tones contribute to the evaluation of alpha-adrenoreceptor activity and the synthesis of endothelial factors. The calculation of MT enables the analysis of the degree of precapillary sphincter closure, which are under the control of the internal myogenic regulatory mechanisms ${ }^{56,57}$ and play the main role in the regulation of the exchange surface area and the number of functioning capillaries.

Thus, the use of this method enables a more detailed analysis of the microcirculatory bed's functional state and its structural and functional units, while also contributing to the evaluation of different processes in microvessels; from analysis of the blood transport function to changes in the dynamics of oxygen utilization by tissues and the identification of factors that have the greatest impact on these processes.

\subsection{Statistical analysis}

All data obtained from the results of the research were checked for normal distribution using the Kolmogorov-Smirnov test and homogeneity of variance using the Levene's test. Analysis of the statistical significance differences of these data was performed using a two-factor ANOVA with repetition (Two-Way Repeated Measures ANOVA). The probability was statistically significant with a $p$-value of less than 0.05 . The choice of this statistical analysis has been associated with the fact that the experimental study has a two-way design (the first factor is phase of the cold pressor test, i.e., $\mathrm{BT} 1, \mathrm{BT} 2, \mathrm{BT} 3$, and the second factor is participant type, i.e., healthy volunteers or rheumatic patients). The use of this test reduces risks of false positives due to multiple testing, which could occur with repeated use of other statistical methods of data processing.

\section{Results and Discussion}

The cold pressor test causes significant changes in microcirculatory-tissue system parameters of the upper limbs. Figure 2 shows examples of registered LDF- and TRO-graphs and their amplitudefrequency spectrums before (Figs. 2(a) and 2(c)) and after (Figs. 2(b) and 2(d)) the cold pressor test.

\subsection{Statistical analysis of groups}

A detailed statistical analysis of the parameter changes for each individual in the two groups displayed a different response of the microcirculatory bed parameters upon the cold exposure, with a different recovery of parameters after CPT. A partial or complete recovery was observed, with individual cases of no recovery of the parameters, which could indicate both the normal state of the microcirculatory bed and the presence of angiospastic disorders. It should be noted that this was a contrast to the statistical analysis of data obtained for the groups of 32 healthy volunteers and 33 patients with diseases of a rheumatological profile. Not individually assessing the volunteers seemed to conceal any significant differences in each basic test.

\subsection{Additional analysis of measured and calculated parameters}

To separate the groups by the criterion of the presence or absence of angiospastic disorders, an additional analysis of the measured and calculated parameters was carried out. The results of analysis showed that for the identity of the possible angoispactic disorders of the microcirculatory bed and related abnormalities, it is necessary to assess changes of the $I_{m}, \mathrm{MT}$ and $\mathrm{OC}$ after CPT.

These parameters were selected because the shift of the balance between vasoconstriction and vasodilation, which occurs under the influence of many factors, leads to a change of functioning of the basic structural and functional units of the microcirculatory bed and decreases blood flow in larger vessels (arteries, arterioles) and their capillary components.

The evaluation of the blood microcirculation index allows conclusions to be made regarding the total decrease of blood flow during these disorders. At the same time, the analysis of changes occurring in the MT and rate of oxygen consumption will give 


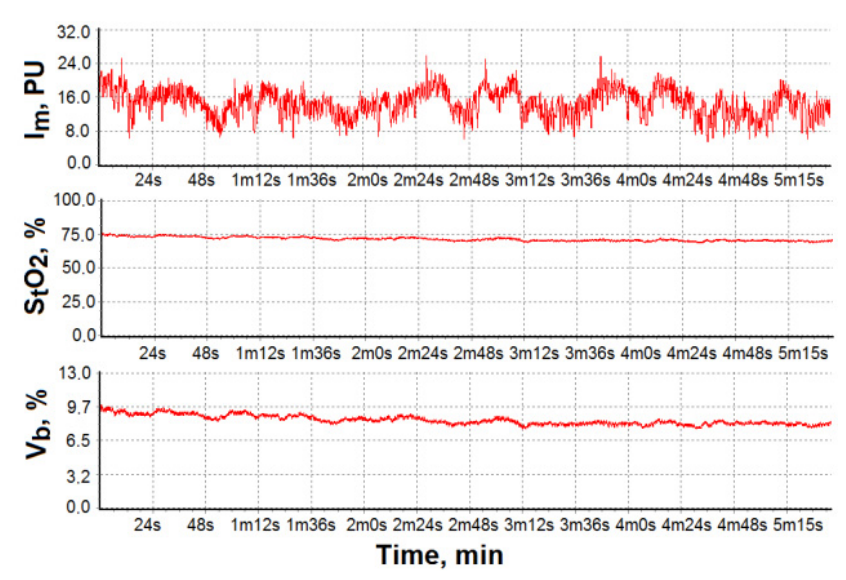

(a)
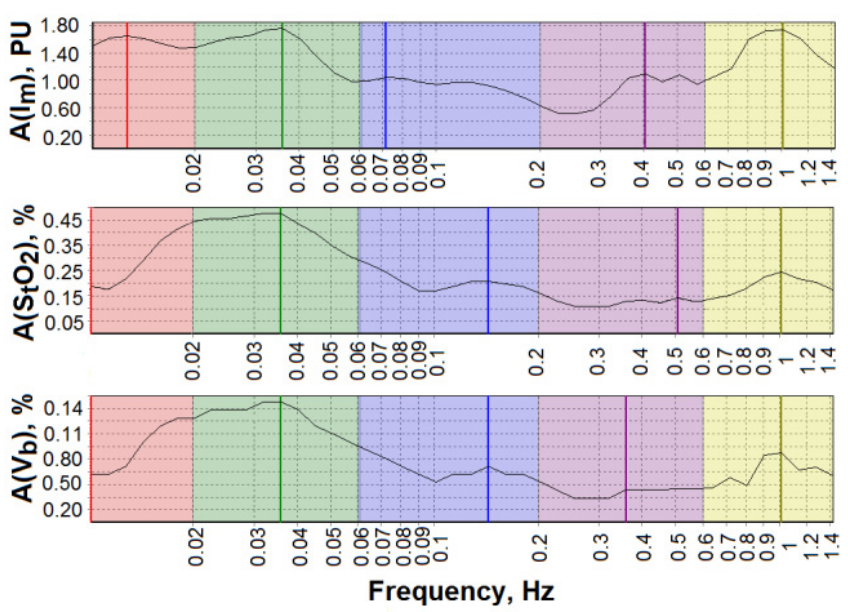

(c)

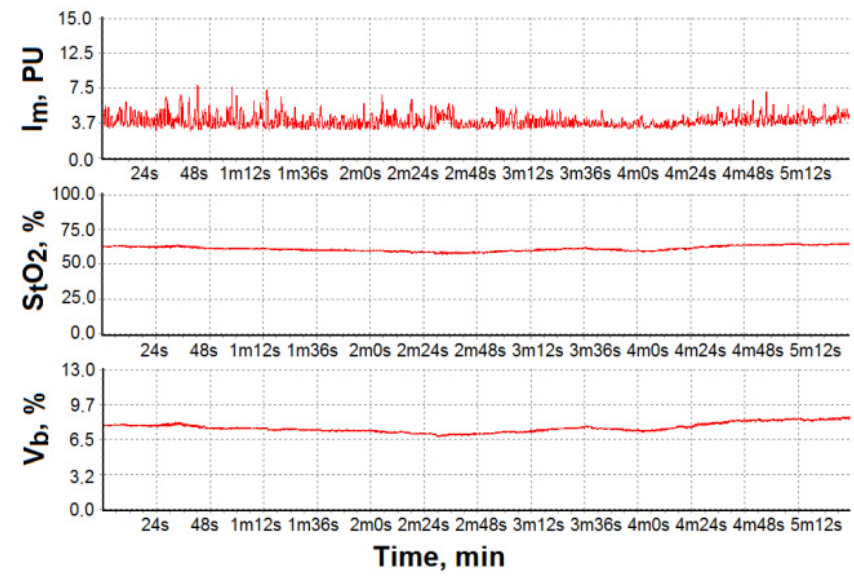

(b)
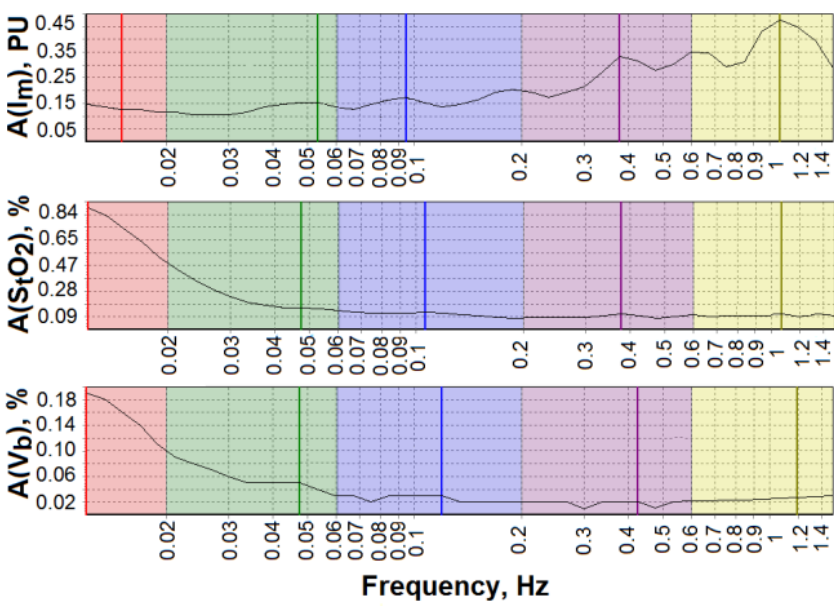

(d)

Notes: $\mathrm{I}_{m}$ — index of blood microcirculation; $\mathrm{StO}_{2}$ - tissue oxygen saturation; $\mathrm{V}_{b}$ - relative blood volume; $\mathrm{A}\left(\mathrm{I}_{m}\right)$ - amplitude of oscillation of index of blood microcirculation, $\mathrm{A}\left(\mathrm{StO}_{2}\right)$ - amplitude of oscillation tissue oxygen saturation; $\mathrm{A}\left(\mathrm{V}_{b}\right)$ - amplitude of oscillation of relative blood volume.

Fig. 2. Examples of registered LDF- and TRO-graphs and their amplitude-frequency spectrums before (a), (c) and after (b), (d) the cold pressor test.

information about the degree of reduction in the number of functioning capillaries, the area of exchange and the decrease in supply and consumption of oxygen to tissues.

The analysis of published data shows that time for stabilization of the all regulatory mechanisms after the cold pressor test can range from 15 min to 30 min depending on the temperature of the influencing factor and cooling time. ${ }^{39}$ At a similar temperature of the cooling factors $\left(15^{\circ} \mathrm{C}\right)$, recovery of those processes should occur within $15-20$ min. ${ }^{58-61}$ The threshold value by which to diagnose angiospastic disorders in this case was from $40 \%$ to $60 \%$ of the initial level. ${ }^{61}$

\subsection{Proposed criteria for identify of angiospastic disorders}

The presence or absence of angiospastic disorders and the possible tendency to angiospasm were suggested based on these new criteria for separation of the diagnostic results. These criteria take into account not only the change of parameters, but also increase (decrease) of the analyzed parameters 
during $\mathrm{CPT}$ in relation to their initial level:

$$
\Delta I_{m}=\left(I_{m 3}-I_{m 2}\right) /\left(I_{m 1}-I_{m 2}\right),
$$

where $I_{m 1}$ is initial value of blood microcirculation index; $I_{m 2}$ is increment of blood microcirculation index after the CPT; $I_{m 3}$ is final value of blood microcirculation index.

$$
\Delta \mathrm{MT}=\left(\mathrm{MT}_{3}-\mathrm{MT}_{2}\right) /\left(\mathrm{MT}_{1}-\mathrm{MT}_{2}\right),
$$

where $\mathrm{MT}_{1}$ is initial value of myogenic tone; $\mathrm{MT}_{2}$ is value of myogenic tone after the $\mathrm{CPT} ; \mathrm{MT}_{3}$ is final value of myogenic tone.

$$
\mathrm{OC}_{3} \geq \mathrm{OC}_{1} \text {. }
$$

where $\mathrm{OC}_{1}$ is initial value of rate of oxygen consumption; $\mathrm{OC}_{3}$ is final value of rate of oxygen consumption.

If the ratio of the final and initial increment $I_{m}$ values relative to the value immediately after the CPT is more than $50 \%$, i.e.,

$$
\Delta I_{m}>50 \%,
$$

and ratio of the final and initial increment $\mathrm{MT}$ values relative to the value immediately after the CPT is more than $50 \%$, i.e.,

$$
\Delta \mathrm{MT}>50 \% \text {, }
$$

and if OC reaches the initial level, then a normal MTS state of limbs/extremities is diagnosed.

If the first two criteria are not met, tendency towards angiospasm is diagnosed. If at least one of the first two criteria is not satisfied, repeat the diagnostic procedure.

To identify the possible reasons for the tendency towards angiospasm, it is necessary to evaluate the amplitude ratios of the cardiac and respiratory blood flow oscillations ${ }^{20}$ :

$$
A_{c}\left(I_{m}\right) / A_{r}\left(I_{m}\right)>1
$$

where $A\left(I_{m}\right)_{c}, A\left(I_{m}\right)_{r}$ are the maximum oscillation amplitudes of cardiac and respiratory origin of the blood microcirculation index.

If 20 min after the CPT, when the first two criteria are not satisfied and the value of the amplitude ratio of the cardiac and respiratory blood flow oscillations is greater than 1 , then we associated the cause of the possible tendency towards angiospasm with increased myogenic tone. If the amplitude ratio value of the cardiac and respiratory oscillations of blood flow is less than or equal to 1 , then we associated the possible cause of angiospasm, in addition to the enhanced MT, with venous stasis. The proposed criteria of the normal state and the tendency to angiospasm, as well as the possible causes of disorders, are presented in Table 2.

It should be noted that the stagnant causes of disorders are almost always combined with high MT. Their detection is a manifestation of the amplification of the myogenic activation, which leads to more significant haemodynamic disorders in the form of venous stagnation. The key distinctive mechanism leading to angiospasm is the myogenic component.

\subsection{Analysis of groups taking into account the proposed criteria}

Using as a criterion for the selection of healthy volunteers without possible microcirculatory bed disorders, the above-described criterion of microcirculatory bed functional state recovery of at least $50 \%$ of the level before the CPT, the following results were obtained

The group of 32 healthy volunteers exhibited two typical responses: in 24 volunteers by the end of the study there was complete parameter recovery, in five volunteers this recovery did not occur. Also a

\begin{tabular}{|c|c|c|c|c|}
\hline No. & Criteria & The normal state & Myogenic reasons & Myogenic-stagnation reasons \\
\hline 1 & $\Delta I_{m}, \%$ & $>50$ & & $<50$ \\
\hline 2 & $\Delta M T, \%$ & $>50$ & & $<50$ \\
\hline 3 & $\mathrm{OC}, \mathrm{AU}$ & $\mathrm{OC}_{3} \geq \mathrm{OC}_{1}$ & & $\mathrm{OC}_{3}<\mathrm{OC}_{1}$ \\
\hline 4 & $A\left(I_{m}\right)_{c} / A\left(I_{m}\right)_{r}, \mathrm{AU}$ & - & $\uparrow(>1)$ & $\downarrow(\leq 1)$ \\
\hline
\end{tabular}
small group of volunteers $(n=3)$ was identified, in which no significant changes in the microcirculatory

Table 2. The proposed criteria of the normal state and the tendency to angispasm, as well as the possible causes of disorders.

The tendency towards angiospasm 
bed parameters occurred throughout the study. As described in this work, ${ }^{62}$ this response is one of the possible responses to cold exposure. The data for these volunteers was also included in the group of volunteers without angiospastic disorders.

Among the 33 patients studied according to the proposed criteria, 18 patients were diagnosed to have a relatively normal functional state of the microcirculatory bed, 15 patients were diagnosed to have a possible tendency to angiospasm. Further, the statistical analysis of the data between the first and second groups in each sample showed significant differences in parameters.

\subsection{Statistical analysis of relatively normal state and state with angiospastic disorders}

To highlight the differences between relatively normal state and presence of angiospastic disorders, detected using the proposed approach, a comparison was conducted. For this a statistical analysis of experimental data was carried out for healthy volunteers without identified angiospastic disorders (relatively normal state), and patients with identified angiospastic disorders.

Figure 3 shows the results of statistical analysis of data from healthy volunteers without identified angiospastic disorders (relatively normal state) (No. 2) and patients with identified angiospastic disorders (No. 2): the index of blood microcirculation (Fig. 3(a)), tissue oxygen saturation (Fig. 3(b)), MT (Fig. 3(c)) and the rate of oxygen consumption (Fig. 3(d)). The result of statistical analysis for all measured and calculation parameters are presented in Table 1, located in the Supplementary material of this paper.

As can be seen from the data after a stimulus in the form of the cold pressor tests, there is a decrease in peripheral blood flow in both groups. The change of the blood flow is due to the development of vascular smooth muscle cell constriction in the vessel wall (arteries and arterioles) as the result of activation of the sympathetic nervous system and alpha-adrenergic receptors. ${ }^{37,63}$ Several studies have indicated the possible participation of endothelial cell vasoconstrictor substances such as endothelin- $1^{35,64}$ in this reaction. Against the background of these processes, increases in MT and decreases in nutritive blood flow were observed in both groups.
Analyzing the changes in these parameters, it is evident that after cold exposure in both the functional states of the microcirculatory bed (with angiospastic disorders and in a relatively normal function state), there is an increase in spasms of precapillary sphincters. As a result of this process, the number of functioning capillaries decreases, which ultimately affects the reduction in capillary blood flow. These processes cause a reduction in tissue oxygen utilization.

It should also be noted that, despite the higher initial level of total blood flow in patients with angiospastic disorders in relation to healthy volunteers without angiospastic disorders, the analysis of shunt blood flow values and the nutritive blood flow between these groups shows significant differences. In healthy volunteers, the greater contribution to the total blood flow is introduced into their capillaries. Patients did not have such a significant difference in the values of these parameters. Additionally, the patients have a lower value in the rate of oxygen consumption. That, in combination with the previously described processes, may indicate initially reduced level in oxygen utilization by tissues. Thus, comparing the parameters between two groups, even conditions of relative rest without provocative impact make the detection of changes in these parameters possible, which may indicate the possible presence of a disorder.

After the termination of cold exposure, the recovery of parameters in the group exhibiting a relatively normal state of microcirculatory bed (without a tendency to angiospasm) and in the group with a tendency to angiospasm occurs in different ways.

In the group with a relatively normal state, after cold influence there is an increase in local temperature and dilatation (widening) of blood vessels, these processes constituting the so-called cold vasodilation reaction. As a result of this reaction, blood vessels lose the ability to shrink, dilate and become a passive vascular bed. ${ }^{65}$ This process may be associated with increased synthesis of endothelium vasodilating substances such as nitric oxide (NO) and other mediators of vasodilation. 20 min after the CPT there is a stabilization of all oscillatory blood flow mechanisms, the decrease of MT and the activation of perfusion. By reducing spasms of precapillary sphincters, the number of functioning capillaries increases and processes of transcapillary oxygen exchange are 


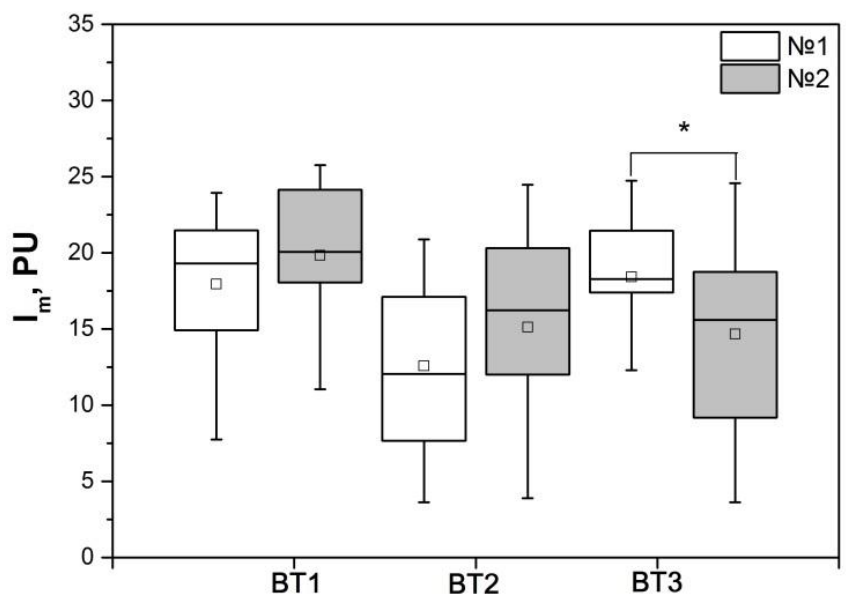

(a)

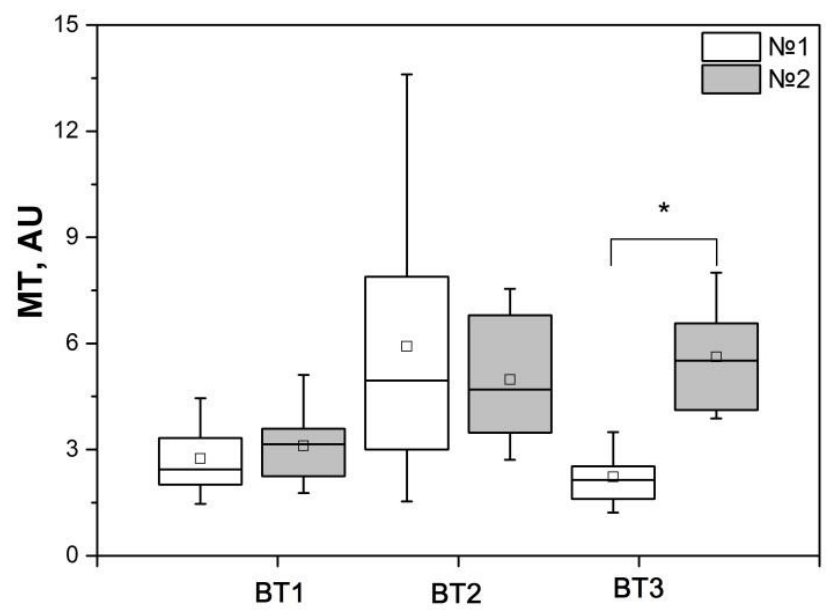

(c)

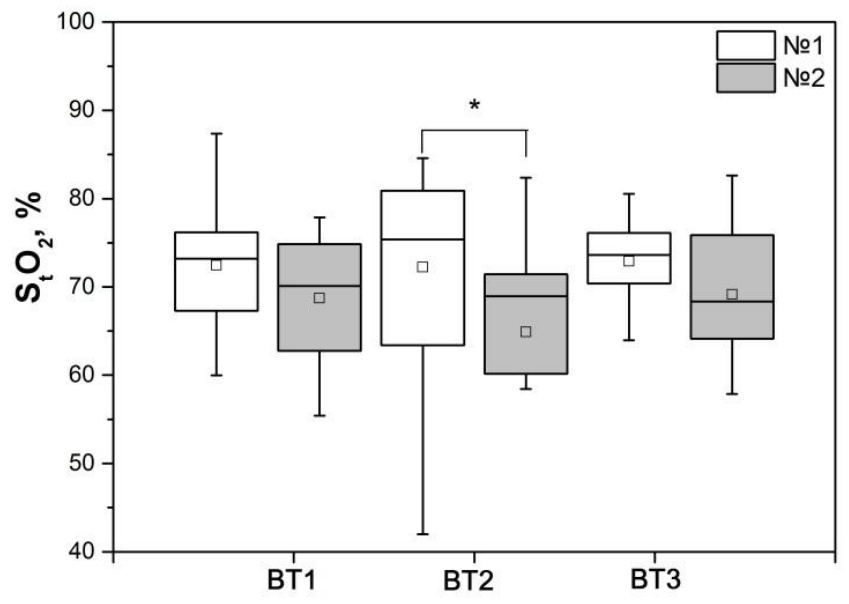

(b)

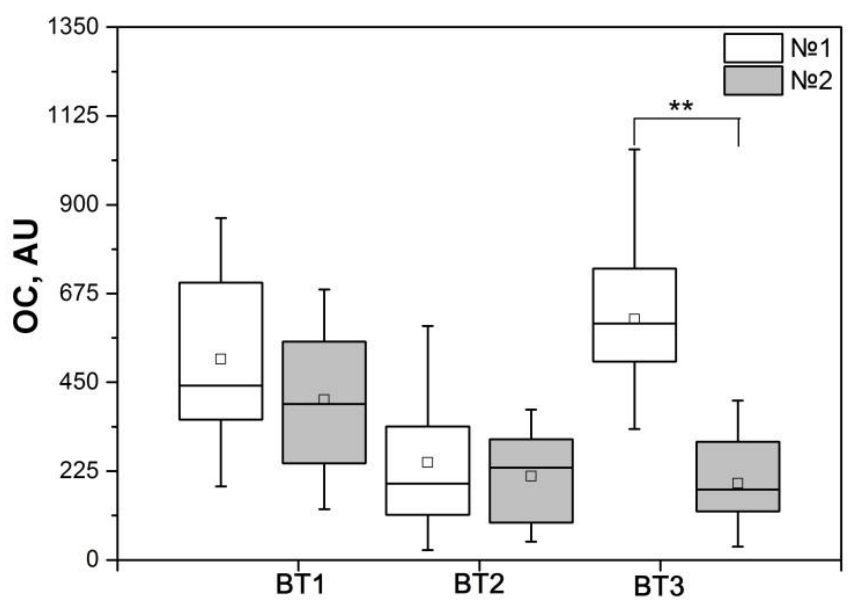

(d)

Notes: $\mathrm{I}_{m}$ — index of blood microcirculation; $\mathrm{StO}_{2}$ — tissue oxygen saturation; MT — myogenic tone; OC — rate of oxygen consumption; BT — basic test Statistically significant differences in the values with ${ }^{*}-p<0.05 ;{ }^{*}{ }_{-} p<0.005$

Fig. 3. The results of static analysis on healthy volunteer without angiospastic disorders (No. 1) and patient with angiospastic disorders (No. 2) data: the index of blood microcirculation (a), tissue oxygen saturation (b), myogenic tone (c) and the rate of oxygen consumption $(\mathrm{d})$.

restored. This is confirmed by the fact that the nutritive blood flow and the rate of oxygen consumption increase.

In the group with a tendency to angiospasm, recovery of microcirculatory bed parameters did not occur. This process may be the result of a shifting balance between vasoconstriction and vasodilatation towards the former, due to intensive and prolonged spasms of the vascular wall and reduced concentration of endogenous vasodilators acting on the endothelium, namely reducing the synthesis of the NO. That may be associated with endothelial dysfunction. ${ }^{33}$ As a result, the value of the total perfusion decrease and MT increases. All these processes lead to an even greater reduction in the number of functioning capillaries, resulting in a decreased proportion of nutritive blood flow and rate of oxygen consumption. Analysis of the possible reasons of angiospastic disorders showed that in most cases they were related to increased MT, with only two patients displaying venous stasis on the background of increased MT.

The additional information about values of analyzed parameters is presented in Table 1 located in the Supplementary material of this paper. 


\subsection{Relationship of angiospastic disorders state of patients with the diagnosis and the results of laboratory studies}

The analysis of the data and their relationship with the diagnosis and the results of laboratory studies have shown that there are several factors, which potentially indicate and confirm the presence of the possible angiospastic disorders in these patients.

Four of the 15 patients identified using the criteria proposed above, had been diagnosed with Raynaud's syndrome. As is known, the basis of Raynaud's syndrome is a local defect in the regulation of vasomotor reactions. Thus, the revealed violation of blood flow and an increase in spasms are only manifestations of the disease. In other patients, the haematologic abnormalities in the form of anaemia $(n=5)$ were observed on background of the main disease. This indicates a decrease of haemoglobin and, consequently, the deterioration of blood flow. Violation of blood flow and angiospasm could provoke and systemic manifestations of rheumatoid disease in the form of vasculitis $(n=3)$, which is characterized by inflammation and destruction of blood vessels. As a result, there is a narrowing of the damaged blood vessels, violation of blood flow and tissue destruction. ${ }^{66}$ The increase of vascular spasms could also be the result of vasodilation disorder and reduction of vasodilatory stimuli against a background of increased sympathetic nervous system activity, which is one of the possible pathogenetic factors of hypertension $(n=8) .{ }^{67}$

It should be noted that some patients had the combined effect of several factors described. Only one patient in the diagnosis (female) did not display factors that could influence the functional state of the microcirculatory bed and cause increased spasms of vessels. Most likely, the detected deviation was due to the short-term increase of vasospastic activity in the background of emotional or hormonal surges, which is typical for females. ${ }^{68}$

\section{Conclusion}

Statistically significant differences between the groups analyzed were found as part of the studies conducted and analysis of data received using methods of laser Doppler flowmetry, tissue reflectance oximetry and pulse oximetry together with the cold pressor test. Wherein, the application of the proposed diagnostic criteria of tendency to angiospasm made it possible to identify subgroups in each group with the presence and absence of angiospastic disorders. In the group of 32 volunteers, 27 people were found to not display any disorders, while in the group of patients, 18 people were observed to have a relatively normal functional state of the microcirculatory bed and 15 people were observed to have a possible tendency to angiospasm. The statistical analysis of experimental data of healthy volunteers without identified angiospastic disorders and patients with identified angiospastic disorders showed that there are significant differences between these states, namely between a relatively normal state and presence of angiospastic disorders. Further analysis of data of these patients identified a relationship between their diagnoses and the results of laboratory studies.

Thus, the evaluation of combined noninvasive optical diagnostic method use, the cold pressor test and proposed diagnostic criteria showed a positive result. This method can be used to detect the presence of possible angiospastic disorders and related complications, as well as microcirculatory bed disorders against the background of other diseases.

Based on the above results, further experimental procedures are planned on a larger volunteer base. This would enable development of more accurate criteria for diagnostically differentiating disorders, i.e., explore the specificity and sensitivity of the methods proposed in identifying the differences in angiospastic disorders present in various diseases. Additionally, this would not only allow to discern the volunteer state of health or disease, but to further improve the ability to detect disease stages and their features. A more in-depth verification of the proposed methodology will also be conducted by associating the study with existing instrumental diagnosis methods.

\section{Acknowledgments}

We would like to thank all of our volunteers and patients for their contribution to this research project.

\section{References}

1. S. Sasaki, and K. Yasuda, Textbook of Angiology: Yasuda Vasospastic Syndrome, Springer-Verlag Publisher (2000). 
2. H. Heidrich, "Functional vascular diseases: Raynaud's syndrome, acrocyanosis and erythromelalgia," Vasa 39(1), 33-41 (2010).

3. C. A. Hitchon, H. S. El-Gabalawy, "Oxidation in rheumatoid arthritis," Arthritis. Res. Ther. 6(6), 265-278 (2004).

4. N. A. Flavahan, "A vascular mechanistic approach to understanding Raynaud phenomenon," Nat. Rev. Rheumatol. 11(3), 146-158 (2015).

5. R. N. Pittman, Regulation of Tissue Oxygenation, San Rafael (CA): Morgan \& Claypool Life Sciences (2011).

6. A. L. Herrick, "Pathogenesis of Raynaud's phenomenon," Rheumatology (Oxford) 44(5), 587-596 (2005).

7. J. A. Block, W. Sequeira, "Raynaud's phenomenon," Lancet 357, 2042-2048 (2001).

8. B. Kahaleh, "Vascular disease in scleroderma: Mechanisms of vascular injury," Rheum. Dis. Clin. North Am. 34(1), 57-71 (2008).

9. F. M. Wigley, "Vascular disease in scleroderma," Clin. Rev. Allergy Immunol. 36(2-3), 150-175 (2009).

10. S. P. Bhatt, R. Handa, G. S. Gulati, S. Sharma, R. M. Pandey, P. Aggarwal, L. Ramakrishnan, S. Shankar, "Peripheral vascular disease in systemic lupus erythematosus," Lupus 16(9), 720-723 (2007).

11. A. H. Kao, J. M. Sabatine, S. Manzi, "Update on vascular disease in systemic lupus erythematosus," Curr. Opin. Rheumatol. 15(5), 519-527 (2003).

12. P. Hartmann, M. Mohokum, P. Schlattmann, "The association of Raynaud's syndrome with rheumatoid arthritis-a meta-analysis," Clin. Rheumatol. 30(8), 1013-1019 (2011).

13. A. Saraux, J. Allain, C. Guedes, D. Baron, P. Youinou, P. Le Goff, "Raynaud's phenomenon in rheumatoid arthritis," Br. J. Rheumatol. 35(8), 752-754 (1996).

14. G. E. Nilsson, T. Tenland, P. A. Oberg, "Evaluation of a laser Doppler flowmeter for measurement of tissue blood flow," IEEE Trans. Biomed. Eng. 27(10), 597-604 (1980).

15. M. J. Leahy, F. F. de Muhl, G. E. Nilsson, R. Maniewski, "Principles and practice of the laserDoppler perfusion technique," Technol Health Care 7(2-3), 143-162 (1999).

16. S. Takatani, P. W. Cheung, E. A. Ernst, "A noninvasive tissue reflectance oximeter. An instrument for measurement of tissue hemoglobin oxygen sarturation in vivo," Ann. Biomed. Eng. 8(1), 1-15 (1980).

17. J. W. Severinghaus, Y. Honda, History of blood gas analysis. VII. Pulse oximetry," J. Clin. Monit. 3(2), 135-138 (1987).
18. A. Stefanovska, M. Bracic, H. D. Kvernmo, "Wavelet analysis of oscillations in the peripheral blood circulation measured by laser Doppler technique," IEEE Trans. Biomed. Eng. 46(10), 12301239 (1999).

19. M. E. Mück-Weymann, H. P. Albrecht, D. Hager, D. Hiller, O. P. Hornstein, R. D. Bauer, "Respiratorydependent laser-Doppler flux motion in different skin areas and its meaning to autonomic nervous control of the vessels of the skin," Microvasc. Res. 52(1), 69-78 (1996).

20. A. I. Krupatkin, "Cardiac and respiratory oscillations of the blood flow in microvessels of the human skin," Hum. Physiol. 34(3), 323-329 (2008).

21. H. Schmid-Schönbein, S. Ziege, R. Grebe, V. Blazek, R. Spielmann, F. Linzenich, "Synergetic interpretation of patterned vasomotor activity in microvascular perfusion: Discrete effects of myogenic and neurogenic vasoconstriction as well as arterial and venous pressure fluctuations," Int. J. Microcirc. 17(6), 346-359 (1997).

22. A. I. Krupatkin, "Blood flow oscillations at a frequency of about $0.1 \mathrm{~Hz}$ in skin microvessels do not reflect the sympathetic regulation of their tone," Hum. Physiol. 35(2), 183-191 (2009).

23. A. I. Krupatkin, "The influence of the sympathetic innervation on the skin microvascular tone and blood flow oscillations," Hum. Physiol. 32(5), 584$592(2006)$.

24. T. Söderström, A. Stefanovska, M. Veber, H. Svensson, "Involvement of sympathetic nerve activity in skin blood flow oscillations in humans," Am. J. Physiol. Heart Circ. Physiol. 284(5), H1638-1646 (2003).

25. H. D. Kvernmo, A. Stefanovska, K. A. Kirkebøen, K. Kvernebo, "Oscillations in the human cutaneous blood perfusion signal modified by endotheliumdependent an endothelium-independent vasodilators," Microvasc. Res. 57(3), 298-309 (1999).

26. P. Kvandal, A. Stefanovska, M. Veber, H. D. Kvernmo, K. A. Kirkebøen, "Regulation of human cutaneous circulation evaluated by laser Doppler flowmetry, iontophoresis, and spectral analysis: Importance of nitric oxide and prostaglandines," Microvasc. Res. 65(3), 160-171 (2003).

27. P. Kvandal, S. A. Landsverk, A. Bernjak, A. Stefanovska, H. D. Kvernmo, K. A. Kirkeboen, "Low-frequency oscillations of the laser Doppler perfusion signal in human skin," Microvasc. Res. 72(3), 120-127 (2006).

28. J. M. Stewart, I. Taneja, M. S. Goligorsky, M. S. Medow, "Noninvasive measure of microvascular nitric oxide function in humans using very lowfrequency cutaneous laser Doppler flow spectra," Microcirculation 14(3), 169-180 (2007). 
29. M. E. Anderson, T. L. Moore, S. Hollis, S. Clark, M. I. Jayson, A. L. Herrick, "Endothelial-dependent vasodilation is impaired in patients with systemic sclerosis, as assessed by low dose iontophoresis," Clin. Exp. Rheumatol. 21, 403 (2003).

30. A. Schlez, M. Kittel, S. Braun, H. M. Hafner, M. Junger, "Endothelium-dependent regulation of cutaneous microcirculation in patients with systemic scleroderma," J. Invest. Dermatol. 120(2), 332-334 (2003).

31. F. Khan, J. J. Belch, "Skin blood flow in patients with systemic sclerosis and Raynaud's phenomenon: Effects of oral L-arginine supplementation," J. Rheumatol. 26, 2389-2394 (1999).

32. R. R. Freedman, R. Girgis, M. D. Mayes, "Abnormal responses to endothelial agonists in Raynaud's phenomenon and scleroderma," J. Rheumatol. 28(1), 119-121 (2001).

33. R. R. Freedman, R. Girgis, M. D. Mayes, "Endothelial and adrenergic dysfunction in Raynaud's phenomenon and scleroderma," J. Rheumatol. 26(11), 2386-2388 (1999).

34. D. S. Celermajer, "Endothelial function: Does it matter? Is it reversible?" J. Am. Coll. Cardiol. 30, 325-333 (1997).

35. D. Abraham, M. Dashwood, "Endothelin-role in vascular disease.," Rheumatology 47, 23-24 (2008).

36. M. R. Zamora, R. F. O'Brien, R. B. Rutherford, J. V. Weil, "Serum endothelin-1 concentrations and cold provocation in primary Raynaud's phenomenon," Lancet 336, 1144-1147 (1990).

37. M. A. Chotani, S. Flavahan, S. Mitra, D. Daunt, N. A. Flavahan, "Silent alpha $(2 \mathrm{C})$-adrenergic receptors enable cold-induced vasoconstriction in cutaneous arteries," Am. J. Physiol. Heart Circ. Physiol. 278(4), H1075-1083 (2000).

38. W. Schady, A. Sheard, A. Hassell, L. Holt, M. Jayson, P. Klimiuk, "Peripheral nerve dysfunction in scleroderma," Q. J. Med. 80, 661-675 (1991).

39. N. Harada, "Cold-stress tests involving finger skin temperature measurement for evaluation of vascular disorders in hand-arm vibration syndrome: Review of the literature," Int. Arch. Occup. Environ. Health 75(1-2), 14-19 (2002).

40. M. A. Greene, A. J. Boltax, G. A. Lustig, E. Rogow, "Circulatory dynamics during the cold pressor test," Am. J. Cardiol. 16, 54-60 (1965).

41. J. Cui, T. E. Wilson C. G. Crandall, "Baroreflex modulation of muscle sympathetic nerve activity during cold pressor test in humans," Am. J. Physiol. Heart Circ. Physiol. 282(5), H1717-1723 (2002).

42. L. Mourot, M. Bouhaddi, J. Regnard, "Effects of the cold pressor test on cardiac autonomic control in normal subjects," Physiol. Res. 58(1), 83-91 (2009).
43. R. G. Victor, W. N. J. Leimbach, D. R. Seals, B. G. Wallin, A. L. Mark, "Effects of the cold pressor test on muscle sympathetic nerve activity in humans," Hypertension 9(5), 429-436 (1987).

44. K. D. Monahan, T. E. Wilson, C. A. Ray, "Omega-3 fatty acid supplementation augments sympathetic nerve activity responses to physiological stressors in humans," Hypertension 44(5), 732-738 (2004).

45. A. B. Lafleche, B. M. Pannier, B. Laloux, M. E. Safar, "Arterial response during cold pressor test in borderline hypertension," Am. J. Physiol. 275 (2 Pt 2), H409-415 (1998).

46. R. D. Gordon, O. Küchel, G. W. Liddle, D. P. Island, "Role of the sympathetic nervous system in regulating renin and aldosterone production in man," J. Clin. Invest. 46(4), 599-605 (1967).

47. M. Pascualy, E. C. Petrie, K. Brodkin, E. R. Peskind, C. W. Wilkinson, M. A. Raskind, "Hypothalamic pituitary adrenocortical and sympathetic nervous system responses to the cold pressor test in Alzheimer's disease," Biol. Psychiatry 48(3), 247-254 (2000).

48. D. Gherghel, S. L. Hosking, I. A. Cunliffe, "Abnormal systemic and ocular vascular response to temperature provocation in primary open-angle glaucoma patients: A case for autonomic failure?" Invest. Ophthalmol. Vis. Sci. 45(10), 3546-3554 (2004).

49. A. Nitenberg, D. Chemla, I. Antony, "Epicardial coronary artery constriction to cold pressor test is predictive of cardiovascular events in hypertensive patients with angiographically normal coronary arteries and without other major coronary risk factor," Atherosclerosis 173(1), 115-123 (2004).

50. A. V. Tankanag, N. K. Chemeris, "Application of the adaptive wavelet transform for analysis of blood flow oscillations in the human skin," Phys. Med. Biol. 53(21), 5967-5976 (2008).

51. A. V. Tankanag, N. K. Chemeris, "A method of adaptive wavelet filtering of the peripheral blood flow oscillations under stationary and nonstationary conditions," Phys. Med. Biol. 54(19), 5935-5948 (2009).

52. E. G. Salerud, T. Tenland, G. E. Nilsson, P. A. Oberg, "Rhythmical variations in human skin blood flow," Int. J. Microcirc. Clin. Exp 2(2), 91-102 (1983).

53. M. Roustit, S. Blaise, C. Millet, J. L. Cracowski, "Reproducibility and methodological issues of skin post-occlusive and thermal hyperemia assessed by single-point laser Doppler flowmetry," Microvasc. Res. 79, 102-108 (2010).

54. A. I. Krupatkin, "Noninvasive estimation of human tissue respiration with wavelet-analysis of oxygen 
saturation and blood flow oscillations in skin microvessels," Hum. Physiol. 38(4), 396-401 (2012).

55. A. V. Dunaev, V. V. Sidorov, A. I. Krupatkin, I. E. Rafailov, S. G. Palmer, N. A. Stewart, S. G. Sokolovski, E. U. Rafailov, "Investigating tissue respiration and skin microhaemocirculation under adaptive changes and the synchronization of blood flow and oxygen saturation rhythms," Physiol. Meas. 35(4), 607-621 (2014).

56. S. S. Segal, "Regulation of blood flow in the microcirculation," Microcirculation 12(1), 33-45 (2005).

57. B. M. Altura, "Chemical and humoral regulation of blood flow through the precapillary sphincter," Microvasc. Res. 3(4), 361-384 (1971).

58. M. J. Corrêa, S. F. Perazzio, L. E. Andrade, C. Kayser, "Quantification of basal digital blood flow and after cold stimulus by laser Doppler imaging in patients with systemic sclerosis," Rev. Bras. Reumatol. 50(2), 128-140 (2010).

59. M. L. Bartelink, H. Wollersheim, E. Leesmans, T. de Boo, T. Thien, "A standardized finger cooling test for Raynaud's phenomenon: Diagnostic value and sex differences," Eur. Heart J. 14(5), 614-622 (1993).

60. J. K. Kristensen, M. Engelhart, T. Nielsen, "Laser Doppler measurement of digital blood flow regulation in normals and in patients with Raynaud's phenomenon," Acta Derm. Venereol. 63, 43-47 (1983).
61. D. O'Reilly, L. Taylor, K. el-Hadidy, M. I. Jayson, "Measurement of cold challenge responses in primary Raynaud's phenomenon and Raynaud's phenomenon associated with systemic sclerosis," Ann. Rheum. Dis. 51, 1193-1196 (1992).

62. S. S. Purkayastha, W. Selvamurthy, G. Ilavazhagan, "Peripheral vascular response to local cold stress of tropical men during sojourn in the Arctic cold region," Jpn. J. Physiol. 42(6), 877-889 (1992).

63. N. Charkoudian, "Skin blood flow in adult human thermoregulation: How it works, when it does not, and why," Mayo Clin. Proc. 78(5), 603-612 (2003).

64. W. Rychlik-Golema, K. Mastej, R. Adamiec, "The role of endothelin-1 and selected cytokines in the pathogenesis of Raynaud's phenomenon associated with systemic connective tissue diseases," Int. Angiol. 25(2), 221-227 (2006).

65. H. A. M. Daanen, "Finger cold-induced vasodilation: A review," Eur. J. Appl. Physiol. 89, 411-426 (2003).

66. M. S. Genta, R. M. Genta, C. Gabay, "Systemic rheumatoid vasculitis: A review," Semin Arthritis Rheum. 36(2), 88-98 (2006).

67. G. Mancia, G. Grassi, C. Giannattasio, G. Seravalle, "Sympathetic activation in the pathogenesis of hypertension and progression of organ damage," $\mathrm{Hy}$ pertension 34(4Pt 2), 724-728 (1999).

68. J. P. Cooke, M. A. Creager, P. J. Osmundson, J. T. Shepherd, "Sex differences in control of cutaneous blood flow," Circulation 82(5), 1607-1615 (1990). 